\title{
Ego Development and Adolescent Academic Achievement
}

\author{
Krisanne Bursik \\ Suffolk University \\ Timothy A. Martin \\ Child \& Family Psychological Services, Inc
}

\begin{abstract}
This study investigated ego developmental differences in adolescent academic orientations and academic achievement. A sample of 142 male and female high school students completed the Washington University Sentence Completion Test and self-report measures assessing academic locus of control, learning orientation (LO), and grade orientation (GO). With increasing ego development, adolescents demonstrated increased internal academic locus of control, stronger LOs, diminished GOs, and higher class rank. Regression analysis indicated that ego level was a significant predictor of academic achievement after controlling for the effects of verbal intelligence and gender. Implications for fostering academic success through theoretically relevant interventions are discussed.
\end{abstract}

Loevinger (1976) conceptualized the ego as a unifying frame of reference that underlies all of our thoughts and actions; ego development is the process of gaining psychological maturity. Viewed as the master trait, ego development encompasses changes in impulse control and character, interpersonal relations, conscious preoccupations, and cognitive style. The model includes nine qualitatively distinct milestones of development, each representing increasingly mature organizations of the self and

Requests for reprints should be sent to Krisanne Bursik, Department of Psychology, Suffolk University, 41 Temple Street, Boston, MA 02114. E-mail: kbursik@suffolk.edu 
the environment. This research extends the theory to the prediction of educational outcomes, examining whether the increasing individual differences in ego development during adolescence are associated with differential modes of approaching academic tasks and varied academic outcomes.

Dispositional constructs associated with academic achievement include academic locus of control, learning orientation (LO), and grade orientation (GO). Students with an internal academic locus of control demonstrate greater academic success (Findley \& Cooper, 1983), as do those with a stronger LO: the degree to which a student is excited by the opportunity to learn, finds intrinsic reward from the learning experience, and feels that knowledge is a reward of its own. Conversely, grade-oriented students base their focus on getting the grade, gearing their approach toward pleasing the instructor in order to gain positive feedback. Research suggests that while a LO predicts academic achievement (Harris \& Harris, 1987), a GO is negatively correlated with academic success (Beck, Rorrer-Woody, \& Pierce, 1991).

Although limited research has demonstrated associations among these constructs, they have not been studied from an ego developmental perspective. In light of the differential timing of maturation for boys and girls, as well as gendered patterns of socialization, we also examined gender differences for these predictors of academic achievement. Further, we explored whether ego level, the organizing and meaningmaking aspect of personality, would emerge as a significant predictor of academic achievement after controlling for the influence of verbal intelligence.

\section{The Stages of Ego Development}

Each stage of ego development is defined and measured by the Washington University Sentence Completion Test (SCT; Loevinger \& Wessler, 1970; Hy \& Loevinger, 1996). The three pre-Conformist stages of ego development are characteristic of infancy and childhood. The emergent ego of the first stage, Presocial Symbiotic, is marked by self-object differentiation; included for theoretical purposes, this stage is not measured by the SCT. During the Impulsive stage the child is focused on the immediate satisfaction of physical needs. Encounters with the environment, especially social interactions, are labeled as strict dichotomies; the child's interpersonal style is receptive, dependent, and egocentric. The Self-Protective stage reflects a first step toward establishing an ability to delay gratification with the introduction of minimal impulse control. The person at this 
stage sees interpersonal relations as exploitative, is often wary and defensive when interacting with others, and has a limited ability to take responsibility for his or her actions. Some adolescents may never mature beyond these early ego stages, but most do.

The Conformist level of ego development includes two stages commonly observed during the adolescent years. Movement into the Conformist stage is marked by the ascendance of identification with the group, adherence to group rules, and acceptance of authority. Gender roles are usually quite conventional, and emotions are clichéd; the Conformist places a high degree of value on appearance, reputation, and social acceptance. The dominant characteristic of the fifth stage, Self-Aware, is that the person has begun to recognize that not everyone, including possibly him/herself, conforms to the previously simple stereotypes. Alternative possibilities are discovered and explored that were previously out of bounds for the Conformist.

Four additional stages of ego development reflect potentials for adult development. The Conscientious stage marks a major shift toward selfevaluated standards, achievement themes, and articulated long-term goals. At the Individualistic stage, the person recognizes him/herself as an individual with a greater ability to differentiate one's own behavior and personality. Tolerance for individual differences continues to evolve, and there is a growing understanding of psychological causation and role differentiation. The final stages, Autonomous and Integrated, are marked by increasing levels of autonomy, morality, and tolerance for ambiguity.

\section{Empirical Research on Ego Development in Adolescence}

Previous research on ego development has supported the developmental nature of the construct; studies indicate that ego stage consistently advances with age during childhood and adolescence (Novy, 1993; Redmore \& Loevinger, 1979). There is also growing evidence that for many, ego development appears to stabilize in young adulthood at the Self-Aware stage (Cohn, 1998). Although variation in ego level has been found to increase dramatically in adolescence (Westenberg \& Gjerde, 1999), significant individual differences in ego development have been found in every age cohort that has been empirically studied.

Other studies have examined gender comparisons in ego development during the adolescent and young adult years. Research suggests that during the high school years both girls and boys cluster around the Conformist and Self-Aware stages, with most of the distribution falling between the Self-Protective (E3) and Conscientious (E6) stages (Gfellner, 
1986; Westenberg \& Gjerde, 1999). Gender differences have been consistently found, with girls demonstrating higher ego development through high school (Cohn, 1991: Gfellner, 1986; Mabry, 1993). Research suggests, however, that by the time children leave adolescence and move into young adulthood, there are no significant differences between the sexes (Bursik, 1995; Redmore, 1983). Based on these studies and the literature documenting gender differences in academic achievement (Matlin, 2000), we sought to explore whether gender moderates the hypothesized relationship between ego development and academic achievement, i.e., that ego development is a stronger predictor for one gender than the other.

Considerable research has also addressed the association of ego development and intelligence, with most studies reporting small to moderate positive correlations (Cohn \& Westenberg, 2004; Cramer, 1999; Hauser, 1976; Westenberg \& Block, 1993). Although the precise relation of these constructs remains unknown, "within any cohort fairly homogeneous with respect to intelligence, one can expect to find a range of character structures" (Loevinger, 1998). Researchers have also reported moderate associations between ego development and vocabulary (Cohn \& Westenberg, 2004; Westenberg, Drewes, Goedhart, Siebelink, \& Treffers, 2004), while simultaneously providing evidence that ego development demonstrates incremental validity after controlling for the influence of verbal skills. Thus, a further aim of this study was to assess the contribution of ego development to understanding individual differences in academic achievement while controlling for the influence of verbal intelligence.

Finally, empirical studies demonstrate that ego development is associated with a number of dispositional variables measuring the adaptive capacities of the individual. Westenberg and Block (1993) found ego development to be associated with increasing degrees of ego resiliency, including the personality constructs of psychological mindedness and intellectualism. Openness to experience (Bursik, 1999; McCrae \& Costa, 1980) and empathy (Carlozzi, Gaa, \& Liberman, 1983) have also been associated with enhanced ego development. Although little empirical research has examined behavioral outcomes, this documented pattern of correlates led us to anticipate positive associations between ego level and several dispositional academic orientations predictive of academic achievement: academic locus of control, LO, and GO.

\section{Academic Locus of Control}

According to Rotter (1954), people's perception of the degree of control they have over their life varies on a continuum from internally based to 
externally based. Individuals with an internal locus of control (internals) believe they have personal control over their life and their destiny. Having an external locus of control (externals) leads to the belief that chance or outside forces determine the fate of the individual. Locus of control is thought to be a relatively stable dispositional characteristic, but one that can be modified somewhat through experience.

The literature examining the association of locus of control and academic achievement demonstrates that internals achieve enhanced school success compared with externals, and that this association is stronger for males than for females (Findley \& Cooper, 1983). Compared with externals, internals feel more able to control outcomes and therefore exert more effort to do so. While internals take pride in their success and feel shame and guilt over failure, externals experience less emotional change with either outcome (Phares, 1976).

More recently, a variety of domain-specific locus of control scales have been developed. The Academic Locus of Control Scale for College Students (Trice, 1985) measures students' beliefs in internal versus external control over academically relevant behaviors. Empirical studies have demonstrated the measure's ability to predict a variety of academic outcomes including grades, attendance, class participation, homework completion, and study time (Ogden \& Trice, 1986; Trice, 1985; Trice, Ogden, Stevens, \& Booth, 1987). In each case, internality was associated with more positive academic outcomes.

\section{Learning Orientation and Grade Orientation}

Students' academic success is clearly affected by the manner in which they approach their academic tasks. Eison (1980) constructed the LOGO to examine two types of orientations: LO and GO. Learning-oriented students are excited by the opportunity to learn and find intrinsic reward from the learning experience. Viewing knowledge as a reward of its own, they are motivated to learn for the sake of learning. Conversely, grade-oriented students base their standards and their focus on getting the grade; they gear their approach to academics toward pleasing the instructor in order to gain positive feedback and external reward.

Research involving the LOGO, and the updated LOGO II (Eison, Pollio, \& Milton, 1982), has revealed interesting findings regarding these orientations and their relations to academic achievement. Milton, Pollio, and Eison (1986) found that students with a stronger LO and a lesser GO were characterized by stronger abilities to reason abstractly, higher 
degrees of personal responsibility, increased self-motivation, and greater interest in new ideas. Further, these students did not believe that luck or fate had a significant effect on their successes; they had strong study habits, positive attitudes towards education, and experienced productive anxiety that promoted test performance. Conversely, grade-oriented students were identified as having a strong desire to behave in a conventional manner and to respect traditional ideas. They also demonstrated both nonproductive test anxiety and poor study habits. Both LO and GO have been associated with SAT scores as well as other tests of academic proficiency such as the American College Test (Harris \& Harris, 1987). Using a college sample, Beck et al. (1991) found that GO was negatively correlated with both grade point average and General Psychology Test scores.

\section{Hypotheses}

Loevinger's theory asserts that during the pre-Conformist and Conformist stages of development the child is highly dependent on external rules of behavior and thought. If the adolescent moves beyond the Conformist stage, self-defined standards become increasingly important. Ego development was hypothesized to predict an increasingly internal locus of control. Further, the shift toward self-evaluated standards, emblematic of the post-Conformist stages, may allow the adolescent to develop a stronger LO, compared with those at Conformist and pre-Conformist ego stages who may be developmentally constricted by their strict adherence to external barometers of success. Thus, we anticipated that students at higher stages of ego development would report a more internal academic locus of control, a stronger LO, and a diminished GO. Further, we hypothesized that with increasing ego development, adolescents of both genders would demonstrate enhanced academic achievement.

\section{METHOD}

\section{Participants}

The sample consisted of 142 students (64 young men and 78 young women) from the public high school of a small, predominantly White, suburban middle class town. Participants included 59 10th graders (83\% of the 10th grade class), 5211 th graders ( $87 \%$ of the 11 th grade class), and 3112 th graders ( $50 \%$ of the 12 th grade class). In all, $74 \%$ of students in these grades 
volunteered to participate in the study. Participants ranged in age from 15 to 19 years (male $M=16.5, \mathrm{SD}=1.1$; female $M=16.2, \mathrm{SD}=.8$ ). The sample included Caucasian (89\%), Hispanic (4\%), African American (1\%), and Asian American (2\%) individuals, with $4 \%$ of the sample not indicating their race.

\section{Procedure}

Participants were recruited for the study with the assistance of the school guidance office. One of the authors visited each English class and gave a brief description of the study to the students. Those students who agreed to participate did so during their regularly scheduled English class. After reading and signing a consent form, each participant completed a packet of measures. One of the primary investigators was present during the data collection to provide instructions and answer any questions. The packet required approximately 1 hour to complete; students were not compensated for their participation.

\section{Measures}

Ego development. Form 81 of the Washington University SCT (Hy \& Loevinger, 1996; Loevinger \& Wessler, 1970) was used to assess ego development. This projective measure instructs the participant to complete 36 incomplete sentences with stems such as Raising a family and Rules are . Each item is individually scored for ego stage and used to derive the continuous item sum score (ISS) and the total protocol rating (TPR) indicating ego stage. The TPR represents the core level of functioning of the individual; it is determined by applying the ogive rules which take into account the total distribution of scores across the 36 stems. A number of studies have demonstrated the measure's robust reliability and construct validity (Cohn \& Westenberg, 2004; Hauser, 1976; 1993; Loevinger, 1979; Redmore \& Waldman, 1975; Westenberg, Hauser, \& Cohn, 2004).

Two raters scored the individual SCT items using instructions from the revised scoring manual (Hy \& Loevinger, 1996). Each item was scored for ego stage; the protocol was then given a TPR using the automatic ogive rules. Prior to coding, the raters participated in a 6-month coding workshop with an expert coder, a coder with previously established inter-rater reliability and familiarity with the measure. During the workshop the raters completed the self-teaching exercises provided in the manual and 
coded protocols from other data sets. Rater 1 established inter-rater reliability with the expert coder with $91 \%$ agreement across the 36 stems and an $87 \%$ agreement for TPR using a coding set of 15 SCT protocols. Rater 2 established inter-rater reliability with the expert coder of $86 \%$ agreement for the 36 stems and 93\% agreement for TPR. In addition, the Spearman correlation coefficient found when the TPRs were compared was .91. Thus, strong inter-rater reliability was achieved prior to coding actual study protocols.

Verbal intelligence. The vocabulary subtest of the Wechsler Intelligence Scale for Children, Third Edition (WISC-III; Wechsler, 1991) has shown the strongest correlation with general intellectual functioning, as measured by the Full Scale IQ on the WISC-III. A paper and pencil version of this subtest was administered as a measure of verbal intelligence. Each item is worth two points and is scored using the WISC-III manual criteria. Possible scores on the measure range from 0 to 60 .

Academic locus of control. A modified version of the Academic Locus of Control Scale for College Students (Trice, 1985) was used. The original measure consisted of 28 true/false items focusing on beliefs in internal versus external control over academically relevant behaviors, such as Grades most often reflect the effort you put into classes. The wording of several items was modified slightly to be more appropriate for high school students, as opposed to college students. Items are coded so that higher scores reflect externality and a lower score reflects a more internal academic locus of control. Trice (1985) provides data supporting the internal consistency of the measure, as well as evidence of its construct validity.

LO/GO. The LOGO II (Eison et al., 1982) was used to assess students' LOs and GOs. The measure consists of 32 items rated on a five-point Likert scale that assess four domains: learning-oriented behaviors ( $I$ do optional reading that my instructors suggest even though I know it will not affect my grade), learning-oriented attitudes (I find the process of learning new material fun), grade-oriented behaviors (I borrow old term papers or speeches from my friends to meet class requirements), and grade-oriented attitudes (Written assignments that are not graded are a waste of student's time). Scores for LO and GO are calculated by summing the scores on the 16 items for both behaviors and attitudes. The authors present evidence demonstrating the internal consistency and test-retest reliability of the LO and GO scales. Subsequent research using the LOGO II to predict 
academic outcomes has also supported the construct validity of the measure (Eison, Pollio, \& Milton, 1986).

Academic achievement. A measure of academic achievement was computed using data on record at the high school. With the permission of the participants and the high school administration, each student's class rank was obtained. At this particular school, class rank is determined on the basis of the students' grade in individual classes with the grade weighted depending on the level of the class. These data were converted to percentile ranks. Class rank was not available for four students who had only recently transferred to the school.

Participants also completed a short demographic questionnaire constructed by the authors. This measure assessed gender, age, race, grade level, and provided data on parental age and educational level.

\section{RESULTS}

\section{Descriptive Analyses}

After scoring each SCT protocol, an ISS and a TPR were generated. The ISSs ranged from 113 to 205 with a mean of 159.73 (SD = 17.31); TPRs ranged from the Impulsive stage (E2) to the Individualistic stage (E7) with a modal rating at the Self-Aware stage (E5). The distribution of TPRs for adolescent boys and girls appears in Table 1. TPRs were used to classify participants into four ego level groups (as in Cramer, 1999): the pre-Con-

TABLE 1

Distribution of Participants by Ego Stage and Gender

\begin{tabular}{lccc}
\hline & \multicolumn{3}{c}{ Gender $(\%)$} \\
\cline { 2 - 4 } Ego Stage & Males & Females & Total \\
\hline Impulsive (E2) & $11(17)$ & $0(0)$ & $11(8)$ \\
Self-Protective (E3) & $14(22)$ & $15(19)$ & $29(20)$ \\
Conformist (E4) & $15(23)$ & $17(22)$ & $32(23)$ \\
Self-Aware (E5) & $16(25)$ & $36(46)$ & $52(37)$ \\
Conscientious (E6) & $8(13)$ & $9(12)$ & $17(12)$ \\
Individualistic (E7) & $0(0)$ & $1(1)$ & $1(<1)$ \\
Total & $64(100)$ & $78(100)$ & $142(100)$ \\
\hline
\end{tabular}

Note. $\times^{2}=18.71, p<.01$. 
TABLE 2

Means, Standard Deviations, and Intercorrelations for All Study Variables

\begin{tabular}{|c|c|c|c|c|c|c|c|c|}
\hline & $M$ & $S D$ & $L O C$ & $L O$ & GO & $E D$ & $I Q$ & $\mathrm{ACH}$ \\
\hline Academic locus of control (LOC) & 14.48 & 3.93 & - & $-.35^{* * * *}$ & $.55^{* * * * *}$ & $-.17^{*}$ & -.08 & $-.19 *$ \\
\hline Learning orientation (LO) & 43.98 & 7.57 & & - & $-.28^{* * *}$ & $.41^{* * * * * *}$ & $.22 * *$ & $.29^{* *}$ \\
\hline Grade orientation (GO) & 47.37 & 7.72 & & & - & $-.30 * * * *$ & -.10 & $-.21 *$ \\
\hline Ego development ISS (ED) & 159.73 & 17.31 & & & & - & $.31 * * * *$ & $=.42 * * * *$ \\
\hline Intelligence (IQ) & 36.93 & 8.92 & & & & & - & $.52^{* * * *}$ \\
\hline Academic achievement $(\mathrm{ACH})$ & $51.86 ?$ & 26.99 & & & & & & - \\
\hline
\end{tabular}

Note. $n$ varies between 138 and 142. ISS, item sum score.

${ }^{*} p<.05 .{ }^{* * *} p<.01 .{ }^{* * * * *} p<.001$.

formist group (Impulsive and Self-Protective stages, $n=40$ ); the Conformist group $(n=32)$; the Self-Aware group $(n=52)$; and the Conscientious group (Conscientious and Individualistic stages, $n=18$ ).

A one-way analysis of variance (ANOVA) on the TPRs indicated a significant gender difference in ego development, $F(1,141)=9.92, p<.01$. Adolescent girls scored on average more than half a stage higher than adolescent boys (male $M=3.90, \mathrm{SD}=1.30$; female $M=4.50, \mathrm{SD}=1.00$ ). Because of this significant difference, all analyses included gender in order to examine possible moderator effects. There was no significant gender difference, however, in verbal intelligence, $F(1,141)=1.31$, NS. Age was not significantly correlated with TPR or ISS, and there was no significant gender difference in age, $F(1,141)=1.68$, NS. Thus, age was not used as a covariate. Means, standard deviations, and intercorrelations of all study variables are presented in Table 2.

\section{Gender and Ego Developmental Differences in Dispositional Academic Orientations}

To examine the predicted developmental differences for these constructs, 2 (gender) $\times 4$ (ego level group) analyses of covariance (ANCOVAs) were performed on the dispositional academic orientations: academic locus of control, LO, and GO. In each analysis, verbal intelligence scores were entered as a covariate.

For academic locus of control, the ANCOVA yielded a significant main effect for gender, $F(1,132)=8.54, p<.01$. Adolescent boys $(M=13.30$, $\mathrm{SD}=.52)$ described a more internal academic locus of control than 
adolescent girls $(M=15.36, \mathrm{SD}=.48)$. The analysis also revealed a significant main effect for ego level group, $F(3,132)=3.31, p<.05$. Post hoc comparisons (Scheffe) indicated that although the means were in the predicted direction, ego group differences failed to reach statistical significance. The Gender $\times$ Ego Group interaction was nonsignificant, $F(3$, $132)=.76$, NS.

The ANCOVA for LO yielded a significant main effect for ego level group, $F(3,132)=7.34, p<.001$. Post hoc comparisons revealed that SelfAware $(p<.01)$ and Conscientious $(p<.001)$ individuals were significantly more learning-oriented than pre-Conformists; the Conscientious group was also significantly higher than the Conformist group $(p<.05)$. The main effect for gender, $F(1,132)=1.43$, and the Gender $\times$ Ego Group interaction $F(3,132)=.23$, were nonsignificant. Similarly, the ANCOVA for GO produced a significant ego level main effect, $F(3,132)=4.49, p<.01$. Students at pre-Conformist levels were significantly more grade-oriented than those at both the Self-Aware and Conscientious ego levels $(p<.05)$. The main effect for gender, $F(1,132)=.97$, and the Gender $\times$ Ego Group interaction, $F(3,132)=.89$, were nonsignificant.

\section{Gender and Ego Developmental Differences in Academic Achievement}

A similar 2 (gender) $\times 4$ (ego level group) ANCOVA was performed on the measure of actual academic achievement (class rank). This ANCOVA yielded a significant main effect for gender, $F(1,129)=4.86, p<.05$. Boys $(M=48.94, \mathrm{SD}=3.10)$ had significantly lower class rank than did girls $(M=58.24, \mathrm{SD}=2.87)$. Significant ego group differences also emerged, $F(3,129)=3.12, p<.05$. Post hoc comparisons revealed that Self-Aware $(p<.05)$ and Conscientious $(p<.01)$ individuals had significantly higher class rank than those at pre-Conformist levels; the Conscientious group was also significantly higher than both the Self-Aware $(p<.05)$ and Conformist groups $(p<.05)$. The Gender $\times$ Ego Group interaction, $F(3$, $129)=.60$, was nonsignificant.

\section{Regression Analysis: Predicting Academic Achievement}

Finally, we performed a regression analysis to examine the relative importance of each of the variables studied and to determine the amount of explained variance associated academic achievement. As seen in Table 3 , the six variables accounted for a significant amount of the variance associated with academic achievement, $R^{2}=.38, F=13.39, p<.001$. In 
TABLE 3

Regression Analysis Predicting Academic Achievement

\begin{tabular}{lrrrr}
\hline Predictor & \multicolumn{1}{c}{ B } & \multicolumn{1}{c}{$S E$} & $\beta$ & \multicolumn{1}{c}{$t$} \\
\hline Verbal intelligence & 1.32 & .22 & $.43^{* *}$ & 5.88 \\
Gender & 9.11 & 4.07 & $.17^{*}$ & 2.24 \\
Ego development (ISS) & .30 & .13 & $.19^{*}$ & 2.28 \\
Academic LOC & -.84 & .62 & -.12 & -1.36 \\
Learning orientation & .13 & .29 & .04 & .45 \\
Grade orientation & -.19 & .31 & -.05 & -.62 \\
Total $R^{2} .38^{* * *}$ & & & & \\
\hline
\end{tabular}

Note. ISS, item sum score; LOC, locus of control. Gender coded 1, male; 2, female. ${ }^{*} p<.05 . * * k<.001$.

addition, verbal intelligence, $\beta=.43, p<.001$, gender, $\beta=.17, p<.05$, and ego development, $\beta=.19, p<.05$, each contributed unique variance associated with academic achievement.

\section{DISCUSSION}

These data clearly demonstrate that even after accounting for gender differences and the role of verbal intelligence, ego development is an important predictor of adolescent academic achievement. Second, the results support the predictable associations among academic locus of control, LO, $\mathrm{GO}$, and ego development. While acknowledging gender similarly in the importance of ego development, gender differences emerged that may be based on the differential maturation and socialization experiences of male and female adolescents.

As with previous studies of general locus of control in adolescence (Kulas, 1996), we observed greater internality among adolescent boys and greater externality among adolescent girls. Somewhat paradoxically, this heightened externality was not associated with lowered academic achievement for girls. Despite socialization patterns that may foster gender differences in locus of control and attribution style (Matlin, 2000), the higher externality among adolescent girls does not appear to be associated with serious academic consequences, at least not at the high school level.

Supporting Loevinger's (1976) view that the ego is a unifying frame of reference, each of the academic orientations showed a significant and 
predictable pattern of change with increasing ego development. Striking developmental differences were particularly evident in participants' responses to the stem Education . Those at the pre-Conformist stages demonstrated their lack of ego development and negativity towards academics with responses such as "is a waste of time," "is a boring thing," and "is garbage," all completions virtually nonexistent at higher ego levels. Conformist adolescents reflected their acceptance of rules, authority, and traditional values with responses including "is very important," "is necessary," and "is great." Movement away from simple clichés and stereotypes was manifested in the sentence completions of Self-Aware adolescents, many of whom responded that education "is the key to getting ahead," "is useful," and "doesn't always come first." At the Conscientious stage, self-evaluated standards and personal values were expressed throughout the protocol and for this particular stem. Responses such as "is important, but it is not something you can read out of a book," and "is a key part of everyone's life, the best foundation one can have going into the world" reflected an increased awareness of the role of education in their lives and futures.

One of the more intriguing findings is the differential pattern of ego development for boys and girls. The distribution of stages by gender indicates greater variability in ego development among adolescent boys compared with girls. Although there were 11 boys scoring at the Impulsive stage, no girls received this TPR. The gender difference in ego development is noteworthy, in light of the association of ego development and academic achievement. What accounts for these gendered patterns of development for boys and girls?

Both biological and social explanations may help to explain the differential patterns of development, particularly at the lower stages of ego development. One explanation may be the different hormonal and physical development that boys and girls experience through puberty. There is evidence that girls go through the process of puberty earlier than boys (Malina, 1990), a process which may promote not only greater physical maturation, but also increased coping skills and psychological maturity for early-maturing girls (Etaugh \& Rathus, 1995). Differential socialization experiences throughout childhood may additionally promote gender-related differences in ego development. From an early age, girls are socialized to value interpersonal relationships to a much greater extent than boys, a pattern that may promote perspective taking skills and enhanced relational development (Bem, 1993). Different socialized patterns of emotional expression, self-conception, and gender role may also play an important role in facilitating or impeding ego development (Bursik, 1995). 


\section{Limitations and Implications for Future Research}

Given these findings, the measurement of ego development provides a useful method for identifying adolescents at risk for academic difficulty. What types of school-based interventions might promote ego development for those at pre-Conformist or Conformist levels? Intervention studies have shown some success in fostering ego development for those below the Self-Aware level (see Cohn, 1998, for a brief review). Individual and group therapy may be a useful vehicle for facilitating the development of those functioning at lower ego levels. Workshops aimed at developing perspective taking skills and fostering tolerance for individual differences would also be relevant, particularly for adolescent boys. These interventions may provide boys with a venue for ego development that mirrors the more naturally occurring cultural and social milieu for adolescent girls. Although we can envision programs to foster the capacities constituting ego development, further research is needed to determine which interventions would best be able to facilitate sustained changes in ego development in adolescence.

Westenberg and Gjerde (1999) argue that development is promoted by frequent interactions with the environment that challenge one's frame of reference; ego development occurs when confronted with information that does not fit existent schemas (Bursik, 1991). Certain social interactions and interpersonal environments may be more or less conducive to ego development in adolescence, in that they serve as environmental pacers. Loevinger (1976) discusses these phenomena as optimally complex stimuli facilitating individual growth. Little is known, however, about the cognitive competencies and accommodative skills needed to process adolescent pacers. Why do some adolescents seek out new learning experiences that others might find daunting? Although Loevinger $(1976,1998)$ asserts that increased intelligence does not necessarily translate into advanced ego development, a central question remains unanswered: Are certain cognitive competencies or styles required in order to reach specific stages in Loevinger's model? For example, is a prerequisite level of openness necessary for novelty, learning, and growth to be positively valued and embraced? This study highlights the need for research designed to better articulate the specific cognitive underpinnings and precursors of this developmental sequence.

Finally, we can only speculate on the implications of these findings for the post-high school experiences of these adolescents. Although research suggests that the gender difference in ego development diminishes throughout the college years (Bursik, 1995; Cohn, 1991), the increased gender similarity in college samples may be a sampling artifact. Adoles- 
cent males functioning at lower stages of ego development may not attend college, and thus data documenting gender similarity in college student ego level may merely be the result of range restriction. This intriguing question has yet to be answered by the empirical research. While crosssectional studies such as this document an academically at-risk group, longitudinal research is needed to empirically demonstrate whether or not these developmental deficiencies continue to predict maladaptive outcomes in later adulthood.

\section{REFERENCES}

Beck, H., Rorrer-Woody, S., \& Pierce, L. (1991). The relations of learning and grade orientations to academic performance. Teaching of Psychology, 18, 35-37.

Bem, S. L. (1993). The lenses of gender. New Haven, CT: Yale University Press.

Bursik, K. (1991). Adaptation to divorce and ego development in adult women. Journal of Personality and Social Psychology, 60, 300-306.

Bursik, K. (1995). Gender-related traits and ego development: Differential patterns for men and women. Sex Roles, 32, 601-615.

Bursik, K. (1999, August). Differential expression of “Big 5" traits with increasing ego development: Paper presented at the Meeting of the American Psychological Association, Boston, MA.

Carlozzi, A., Gaa, J., \& Liberman, D. (1983). Empathy and ego development. Journal of Counseling Psychology, 30, 113-116.

Cohn, L. (1991). Sex differences in the course of personality development: A meta-analysis. Psychological Bulletin, 109, 252-266.

Cohn, L. (1998). Age trends in personality development: A quantitative review. In P. M. Westenberg, A. Blasi, \& L. Cohn (Eds.), Personality development: Theoretical, empirical, and clinical investigations of Loevinger's conception of ego development (pp. 133-143). Mahwah, NJ: Erlbaum.

Cohn, L., \& Westenberg, P. M. (2004). Intelligence and maturity: Meta-analytic evidence for the incremental and discriminant validity of Loevinger's measure of ego development. Journal of Personality and Social Psychology, 86, 760-772.

Cramer, P. (1999). Ego functions and ego development: Defense mechanisms and intelligence as predictors of ego level. Journal of Personality, 67, 735-760.

Eison, J. (1980). The development and validation of a scale to assess differing student orientations towards grades and learning. Dissertation Abstracts International, 40, 4460.

Eison, J., Pollio, H., \& Milton, O. (1982). LOGO II: A user's manual. Knoxville: The University of Tennessee, Learning Resource Center.

Eison, J., Pollio, H., \& Milton, O. (1986). Educational and personal characteristics of four different types of learning- and grade-oriented students. Contemporary Educational Psychology, 11, 54-67.

Etaugh, C., \& Rathus, S. (1995). The world of children. Fort Worth, TX: Harcourt Brace.

Findley, M., \& Cooper, H. (1983). Locus of control and academic achievement: A literature review. Journal of Personality and Social Psychology, 44, 419-427.

Gfellner, B. (1986). Changes in ego and moral development in adolescents: A longitudinal study. Journal of Adolescence, 9, 281-302. 
Harris, C., \& Harris, J. (1987). Learning orientation and academic achievement: Paper presented at the Annual Meeting of the Southeastern Psychological Association, Atlanta, GA.

Hauser, S. (1976). Loevinger's model and measure of ego development. Psychological Bulletin, 83, 928-955.

Hauser, S. (1993). Loevinger's model and measure of ego development: A critical review II. Psychological Inquiry, 4, 23-30.

Hy, L., \& Loevinger, J. (1996). Measuring ego development (2nd ed.). Mawah, NJ: Erlbaum.

Kulas, H. (1996). Locus of control in adolescence: A longitudinal study. Adolescence, 31, 721-729.

Loevinger, J. (1976). Ego development. San Francisco: Jossey-Bass Publishers.

Loevinger, J. (1979). Construct validity of the sentence completion test of ego development. Applied Psychological Measurement, 3, 281-311.

Loevinger, J. (1998). Completing a life sentence. In P. M. Westenberg, A. Blasi, \& L. Cohn (Eds.), Personality development: Theoretical, empirical, and clinical investigations of Loevinger's conception of ego development (pp. 347-354). Mahwah, NJ: Erlbaum.

Loevinger, J., \& Wessler, R. (1970). Measuring ego development I: Construction and use of the Sentence Completion Test. San Francisco: Jossey-Bass.

Mabry, C. (1993). Gender differences in ego level. Psychological Reports, 72, 752-754.

Malina, R. (1990). Physical growth and performance during the transition years. In R. Montemayor, G. R. Adams, \& T. P. Gullota (Eds.), From childhood to adolescence: A transitional period? (pp. 41-62). Newbury Park, CA: Sage.

Matlin, M. (2000). The psychology of women. Fort Worth, TX: Harcourt Brace.

McCrae, R., \& Costa, P. (1980). Openness to experience and ego level in Loevinger's sentence completion test: Dispositional contributions to developmental models of personality. Journal of Personality and Social Psychology, 29, 1179-1190.

Milton, O., Pollio, H., \& Eison, J. (1986). Making sense of college grades. San Francisco, CA: Jossey-Bass.

Novy, D. (1993). An investigation of the progressive sequence of ego development levels. Journal of Clinical Psychology, 49, 332-338.

Ogden, E., \& Trice, A. (1986). The predictive validity of the academic locus of control scale for college students: Freshman outcomes. Journal of Social Behavior and Personality, 1, 649-652.

Phares, E. (1976). Locus of control in personality. Morristown, NJ: General Learning Press.

Redmore, C. (1983). Ego development in the college years: Two longitudinal studies. Journal of Youth and Adolescence, 12, 301-306.

Redmore, C., \& Loevinger, J. (1979). Ego development in adolescence: Longitudinal studies. Journal Youth and Adolescence, 8, 1-20.

Redmore, C., \& Waldman, K. (1975). Reliability of a sentence completion measure of ego development. Journal of Personality Assessment, 39, 236-243.

Rotter, J. (1954). Social learning and clinical psychology. Englewood Cliffs, NJ: Prentice-Hall.

Trice, A. (1985). An academic locus of control scale for college students. Perceptual and Motor Skills, 61, 1043-1046.

Trice, A., Ogden, E., Stevens, W., \& Booth, J. (1987). Concurrent validity of the academic locus of control scale. Educational and Psychological Measurement, 47, 483-486.

Wechsler, D. (1991). Wechsler Intelligence Scale for Children (3rd ed.). Psychological Corporation, San Antonio: Harcourt Brace \& Company.

Westenberg, P. M., \& Block, J. (1993). Ego development and individual differences in personality. Journal of Personality and Social Psychology, 65, 792-800. 
Westenberg, P. M., Drewes, M. J., Goedhart, A. W., Siebelink, B. M., \& Treffers, D. A. (2004). A developmental analysis of self reported fears in late childhood through mid adolescence: Social-evaluative fears on the rise. Journal of Child Psychology and Psychiatry, $45,481-495$.

Westenberg, P. M., \& Gjerde, P. F. (1999). Ego development during the transition from adolescence to young adulthood: A 9-year longitudinal study. Journal of Research in Personality, 33, 233-252.

Westenberg, P. M., Hauser, S. T., \& Cohn, L. (2004). Sentence completion measurement of personality development. In M. J. Hilsenroth (Ed.), Comprehensive handbook of psychological assessment, Vol. 2: Personality Assessment_Projective. New York: John Wiley. 
Copyright (C) 2006, Society for Research on Adolescence 